
Modeling

Vol. 2, No. 1, pp. 46-54, 2021

\title{
Wireless Chaos-Based Communication System: Literature Review
}

\author{
Siti Hadiaty Yuningsih ${ }^{1 *}$, Sudradjat $^{2}$, Sukono $^{3}$, Subiyanto $^{4}$ \\ ${ }^{1,2,3}$ Departement of Mathematics, Faculty of Mathematics and Natural Science, \\ Universitas Padjadjaran, Indonesia \\ ${ }^{4}$ Departement of Marine Science, Faculty of Fishery and Marine Sciencee, \\ Universitas Padjadjaran, Indonesia
}

*Corresponding author email: siti20025@mail.unpad.ac.id

\begin{abstract}
Since the early 1990s, a slew of chaotic-based communication systems have been proposed, all of which take advantage of chaotic wave form properties. The inspiration stems from the substantial benefits that this form of nonlinear signal offers. Many communication schemes and applications have been specifically designed for chaos-based communication systems to achieve this goal, with energy, data rate, and synchronization awareness being taken into account in most designs. However, non-coherent chaos-based systems have recently received a lot of attention in order to take advantage of the benefits of chaotic signals and noncoherent detection while avoiding the use of chaotic synchronization, which has poor performance in the presence of additive noise. This paper provides a thorough examination of all wireless radio frequency chaos-based communication systems. It begins by describing the difficulties of chaos implementations and synchronization processes, then moves on to a thorough literature review and study of chaos-based coherent techniques and their applications.
\end{abstract}

Keywords: Chaos-based communication systems, chaos implementation, chaotic synchronization

\section{Introduction}

Shannon's 1947 realization that a noise-like signal with a waveform of maximum entropy results in an optimized channel potential in communications (Shannon, 1998) and implementation of a functional chaotic electrical circuit have sparked a lot of interest in the field of chaos-based communication systems (Chua, 1980). In general, chaotic maps have the ability to produce an infinite number of low cross-correlated signals due to their high sensitivity to initial conditions. Chaotic signals have proven to be a natural candidate for multi-user spread-spectrum modulation schemes due to their wideband characteristics (Lau \& Tse, 2003; Kaddoum et al., 2013). There is an information theoretic study of the capacity of chaos in digital communication systems, emphasizing that there is no underlying concept prohibiting the use of chaos in digital communications (Hasler \& Schimming, 2002). Some of these chaosbased modulations provide similar benefits to traditional spread-spectrum modulations, such as fading mitigation in time-varying channels (Xia et al., 2004), jamming resistance combined with low probability of interception (LPI) (Yu \& Yao, 2005), and safe communications (Lynnyk \& Elikovský, 2010). In multi-user spread-spectrum communication systems, several studies have been done on reducing multi-user interference and the peak-to-average power ratio (PAPR), showing that chaos-based sequences outperform Gold and many other independent, identically distributed sequences (Vitali et al., 2006; Mazzini et al., 1997).

There have been two groups of chaos-based communication systems suggested and studied: Coherent identification in chaos-based communication systems: In these schemes, a synchronized copy of the chaotic signal is 
produced at the receiver, and the transmitted data is recovered by exploiting this replica in various methods (Kolumbán et al., 1997).

Chaos-based communication systems with non-coherent detection: Data recovery is accomplished in these schemes by detecting features in the received signal without the use of channel state information or the regeneration of any local chaotic signal at the receiver (Lau \& Tse, 2003; Kennedy et al., 2000).

Demodulation is a significant challenge for chaotic communications with coherent receivers because certain chaotic synchronization models perform poorly in noisy environments (Lau \& Tse, 2003; Kolumbán et al., 1996). Non-coherent receivers, such as differential chaos shift keying (DCSK) systems or chaos-based on off Keying (COOK) systems, on the other hand, do not require chaotic signal generation and synchronization on the receiver side to recover the transmitted data (Lau \& Tse, 2003; Kis et al., 1998). Non-coherent receivers, such as differential chaos shift keying (DCSK) systems or chaos-based on off Keying (COOK) systems, on the other hand, do not require chaotic signal generation and synchronization on the receiver side to recover the transmitted data (Lau \& Tse, 2003; Kis et al., 1998). As a result of this significant advantage, an increasing amount of research has been done in this field, with several novel non-coherent chaos-based communication systems being proposed. As a result of this significant advantage, an increasing amount of research has been done in this field, with several novel non-coherent chaos-based communication systems being proposed.

\section{Chaotic Signal Generation, Noise Cleaning and Synchronization Methods}

\subsection{Chaotic Maps Implementation}

Many common chaotic maps have been developed and used in many applications since the 1980s, including the Chebyshev polynomial function (CPF) map, the piece wise linear (PWL) chaotic map, the chaotic Markov map, the tent map, and others (Silva \& Young, 2000). Since such systems remember their history, they are referred to as dynamical systems. For example, 1D discrete-time autonomous chaotic dynamical systems can be defined using a mapping function $f: S \rightarrow S$ (where $S$ denotes the state space) and evaluated using a defining vector field and the initial condition $x_{0}$, as shown below.

$$
x_{k+1}=f\left(x_{k}, \mu\right): x \in S \subseteq R^{K}
$$

where $x_{k}$ is the state variable sampled at the $K^{t h}$ sampling moment, $f($.$) is the iterative function that defines the$ system dynamics, and $\mu$ is the vector of the system dynamics parameters.

The discrete-time representation of dynamical systems will easily provide us with an understandable perspective on the generation of chaotic signals. To put it another way, discrete-time representations make it easier to analyze a dynamical system using an iterative function whose output is a new set of state variables and the current set of state variables as its input. The intervals at which the state variables are modified are determined by the sampling time. Continuous dynamic systems, on the other hand, such as differential equations (Devaney, 2003; Alligood et al., 1996; Sambas et al., 2020; Mobayen et al., 2021), produce continuous (i.e. analog) chaotic signals with theoretically unknown intervals. Chua's circuit, for example, is commonly used to produce an analogue chaotic signal (Chua \& Lin, 1990).

Non-linear dynamical systems can potentially generate an infinite number of uncorrelated chaotic signals by using slightly different initial values in the same system due to their unique properties and responsive dependency on initial conditions. This means that two nearby starting initial conditions will result in two state variable trajectories that are completely uncorrelated (Devaney, 2003). As a result, in order to resolve the major disadvantages of quantization while keeping the number of bits low, a strict technique is needed to boost the quality of the chaotic signal while still ensuring a high degree of protection and randomness (Delgado-Restituto \& Rodriguez-Vazquez, 2001).

Many implementation methods to improve the randomness efficiency of chaotic signals and increase their periodic loop have been proposed in the literature (Delgado-Restituto \& Rodriguez-Vazquez, 2001; Wu \& Baleanu, 2014). Giard et al., (2012) investigates and evaluates the application of discrete-time chaotic generators. This research focuses on the power consumption, resource use, and maximum execution frequency of two common Field Programmable Gate Array implementations (Sambas et al. 2021; Vaidyanathan et al., 2018; Sambas et al., 2019). Reverse-time chaos is used in (Bailey et al., 2014) to create hardware chaotic systems that run at speeds comparable to current state-of-the-art while requiring much less complex circuitry. Nejati et al. (2012) and Beirami et al. (2012) investigate an alternative method for generating chaotic signals with strong randomness properties and low complexity. In their paper, they present the zigzag map, a novel discrete chaotic map with excellent chaotic behavior. Two circuit implementations based on the switched current technique and current-mode affine interpolation of the breakpoints are used to implement this map. 


\subsection{Noise Cleaning for Chaotic Signals}

Chaotic signals are distorted by additive noise when used in wireless communication systems. As a result, the signal-to-noise ratio must be improved to improve the efficiency of chaos-based modulation schemes. Furthermore, since chaotic signals behave like noise, linear filters are no longer useful for this role. Furthermore, removing chaotic time series from a mixture with a noise variable or denoising chaotic time series is a difficult open problem. As a result, the output of any noise cleaning algorithm is highly influenced by the amount of a priori data available.

Many approaches to reducing or cleaning the noise present in this class of non-linear signals are suggested, based on a priori knowledge of chaotic dynamic systems. To estimate the states of noisy chaotic samples, Luca et al. (2006) and Soriano et al. (2011) proposed an initial discrete-time Kalman filtering implementation. In fact, Kalman filtering is a well-known state-space estimator for linear systems with Gaussian noise that provides recursive minimum meansquared error estimates. In addition, the latter cannot be used to estimate non-linear state space models directly. As a result, an approximate solution is needed, allowing the kalman filtering system to be extended to nonlinear state-space models. In a similar vein, adaptive signal processing methods are proposed in (Chen et al., 2014) to minimize noise and improve overall communication system efficiency. As a result, the filtering method that makes use of the a priori chaotic map $f\left(\right.$ i. e. $\left.x_{k+1}=f\left(x_{k}\right)\right)$ is viewed as an optimization problem with a cost function $C$ of the form

$$
C(\hat{x}, r)=c_{1}(\hat{x}, r)+\Gamma c_{2}(\hat{x})
$$

is held to a minimum (Lee, 1997). The function $c_{1}$ calculates the distance between the estimated (i.e. filtered) point $x_{k}$ and the noisy obtained point, the constraint function $c_{1}$ indicates how well the estimated orbit $t s$ is estimated, and the weight function 0 is the weight function. Iterative methods called method I and method II suggested in (Lee \& Williams, 1997) are used to find the best solution for equation (2).

\subsection{Synchronization Methods}

Synchronization is an important step in creating cohesive communication schemes. Many synchronization methods for chaos-based communication systems have been suggested in the literature. We will briefly summarize these strategies in this survey to give readers an idea of the difficulty in designing coherent chaos-based communication systems. As a result, we can divide these approaches into two categories: 1) Methods for synchronizing chaos (Pecora \& Carroll, 1990; Yang et al., 2012). 2) Application of traditional synchronization methods to chaos-based communication systems.

Master and slave systems are used in the first type of synchronization, in which a coupling chaotic signal transmitted from the master device is used to drive the slave system, causing it to produce an exact synchronized copy of the coupling signal (Vaidyanathan et al., 2019; Vaidyanathan et al., 2020; Vaidyanathan., 2021). The second type of synchronization ranges from phase and sampling synchronization in wireless communication systems to chaosbased coherent communication systems. The chaotic generator on the receiver side must have the same initial condition as the chaotic generator on the transmitter side in this category of synchronization. The aim of these synchronization techniques is to eliminate clock drift at the receiver so that a synchronized chaotic sequence can be produced. These methods, as well as recent work in these fields, will be presented in the subsections that follow.

\subsection{Chaos Synchronization}

The chaotic synchronization at the receiver side is a major challenge for coherent chaos-based communication systems. Many chaotic synchronization methods have been suggested in the literature to accomplish this goal. Yamada and Fujisaka were the first to investigate this phenomenon in (Fujisaka \& Yamada, 1983; Afraimovich et al., 1986). When Pecora and Carroll presented their chaotic synchronization system and proposed its application to secure communications (Pecora \& Carroll, 1990), this subject sparked a lot of interest. A master and a slave system are needed in the proposed method, with a single signal from the master system driving the slave system (Pecora \& Carroll,1990; Cuomo \& Oppenheim, 1993).

As a result, a traditional master-slave system is formed by two chaotic systems. The same set of differential equations, with the same parameter values, describe both systems. Pecora \& Carroll (1990) demonstrated that chaotic synchronization occurs when the performance of at least one of the first chaotic systems coupled differential equations is accessible to the second chaotic system. As a result, the time-series signal provided by one of the chaotic systems differential equations is said to drive the other chaotic system. The master chaotic system is referred to as the master system, while the slave chaotic system is referred to as the slave system. Suykens et al. (1997) and Yalçin et al. 
(2001) both looked at the idea of a master-slave synchronization system. Table 1 summarizes the forms that have been defined in the following way.

Table1. Different driving types

\begin{tabular}{ll}
\hline Driving type & Description \\
\hline Directional driving & $\begin{array}{l}\text { One chaotic system (generator) is used as the source of driving } \\
\text { transmitting one or more driving signals to the other generators } \\
\text { two chaotic systems are coupled and driven with each other in a } \\
\text { mutual way (Zhou \& Roy, 2007; Sambas et al., 2015) }\end{array}$ \\
Bidirectional driving & $\begin{array}{l}\text { Many chaotic systems are couple with each other, this is named a } \\
\text { complex dynamic network (Li \& Chen, 2006) } \\
\text { External signals drive the chaotic system present in a network to } \\
\text { External driving }\end{array}$ \\
\hline
\end{tabular}

One or more driving signals are transmitted from the master system (transmitter) to the slave system (receiver) via the communication channel to synchronize the slave system in the communication area. This mechanism causes chaos synchronization by forcing the slave system to obey the master system by producing an exact copy of the transmitted signal (driving signal). The proposed chaos synchronization methods in (Mahmoud, 2014; Wu et al., 2014) can be summarized as shown in Table 2.

Table 2. Chaos synchronization methods

\begin{tabular}{ll}
\hline Sync. Method & Description \\
\hline Complete synchronization & $\begin{array}{l}\text { Also called identical synchronization. In complete } \\
\text { synchronization a complete agreement of the } \\
\text { trajectories of the master and slave system exists. }\end{array}$ \\
Generalized form of complete synchronization, for \\
which the slave system trajectory converges to the \\
master's one in the sense of a one-to-one mapping f \\
(Mbe et al., 2014) \\
A special case of generalized synchronization with \\
the one-to-one mapping involved being a simple \\
linear function $f()=a$ (Yu et al., 2014) \\
The slave system converges to the same phase of the \\
master system, though their trajectories are not the \\
same \\
Phase synchronization \\
A time-delayed version of complete synchronization \\
for which the slave system coincides with the time- \\
delayed dynamics of the master system
\end{tabular}

\subsection{Chaos-Based Coherent Modulation Schemes}

According to the form of reception used, chaos-based communication systems are divided into two categories: coherent and non-coherent (Vali et al., 2010; Fang et al., 2013; Jovic, 2015). We will present various coherent modulation designs in this section, as well as recent work on each of these systems. Several methods for encoding data using chaotic signals have been introduced. A chaotic series and a low-level data signal combined in an additive way.

\subsubsection{Coherent Analog Modulations}

\subsubsection{Chaotic Masking}

In the analog domain, chaotic masking (Cuomo et al., 1993) is the most commonly used and researched modulation. The analog signal (information) $s(t)$ is applied to the transmitted chaotic signal $x$, as shown in Figure 1 (t). The receiver reconstructs the original chaotic signal thanks to chaos synchronization, and the analog information 
is retrieved by subtracting the replicated chaotic signal from the incoming signal. Furthermore, Milanovic \& Zaghloul (1996) and Liao \& Huang (1999) address other methods that have been proposed to enhance chaotic masking modulation.

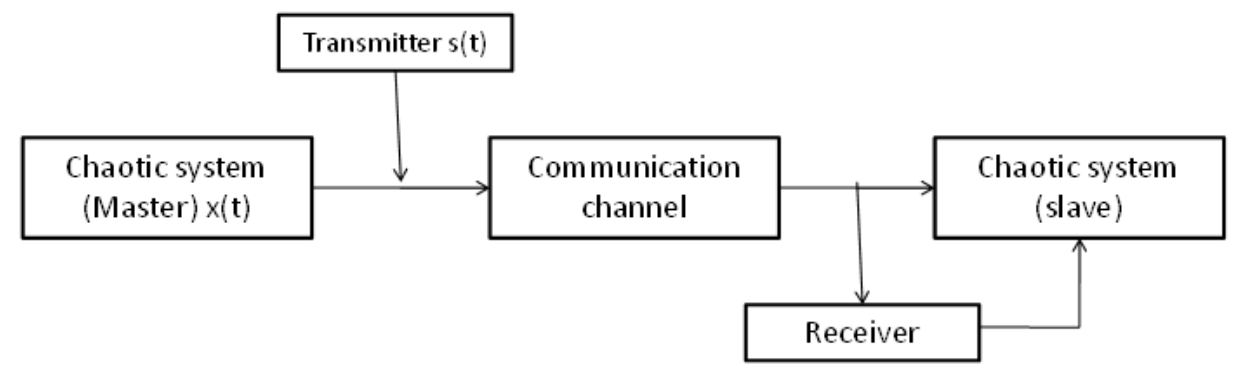

Figure 1. Chaotic masking system model

Against some threats, the security aspects of such modulation are dubious. In reality, the transmitted signal can provide useful information to an intruder, allowing him to build the master systems dynamics. Furthermore, adding the message $s(t)$ to the chaotic signal $x(t)$ causes the resulting signal to shift in force. As a result, an attacker can estimate the transmitted data by observing the transmitted signal's strength. As a result, without modifying the encryption structure, reducing the strength of the message $s(t)$ in comparison to the driving signal cannot effectively eradicate this security flaw.

\subsubsection{Chaotic Modulation}

In chaotic modulation, the analog information is inserted directly into the chaotic system to alternate its dynamic model, rather than applying the message $s(t)$ to the chaotic signal as in chaotic masking. As a result, the analog information is contained in the chaotic signal produced.

At the receiver end, the receiver senses a shift in the chaotic signals complex behavior and recovers the analog data (Feng \& Tse, 2001).

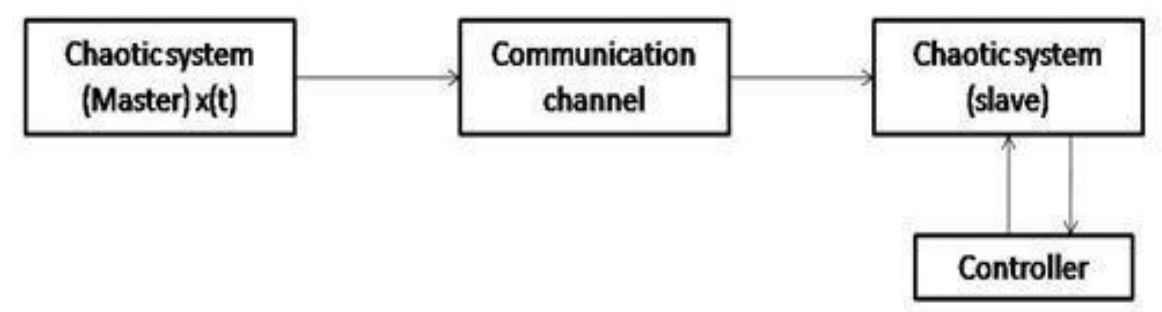

Figure 2: Chaotic modulation system model

Chaotic modulation schemes, in comparison to chaotic masking schemes, can precisely recover the message signal $s(t)$ (in an asymptotical manner) if certain conditions are met and can outperform chaotic switching systems. Furthermore, if the chaotic system is well-designed, the chaotic modulation technique can be used to send several message signals at the same time $(t)$. As a result, the technique's main drawback is its reliance on the controller, as well as the requirement that each chaotic system have a unique controller design. Furthermore, the controllers do not even exist in certain situations, which is a significant downside for this technique (Feng \& Tse, 2001; Chen et al., 2014).

\subsubsection{Chaos-Based Non-Coherent Modulation Schemes}

The majority of chaos communication schemes with coherent receivers are still in their early stages of creation and evolution, facing numerous challenges. When it comes to coherent detection, reliable synchronization between the chaotic structures at the transmitter and receiver is critical, since it necessitates the presence of coordinated replicas of chaotic signals at the receiver When it comes to low signal-to-noise conditions, the communication systems applied chaos synchronization scheme is critical, as coherent detection has proven to be difficult in low SNR situations.

Coherent communications, on the other hand, are the state of the art in the wireless communications domain. To demodulate the received information without error, coherent receivers need perfect knowledge of channel state information at the receiver side. Training-based channel estimation methods using pilots are used to achieve this goal, as is the case with existing long-term evolution (LTE) systems. Such estimation methods, on the other hand, result in 
a substantial increase in receiver complexity as well as signaling overhead. In slow-fading networks, on the other hand, the transmitter will constantly send pilot symbols, resulting in the waste of certain resources due to unnecessary pilot transmissions. Another big drawback of coherent communications is that they degrade when channel estimation errors occur, which is particularly noticeable when there is a lot of movement. As a result, several research groups in the field of chaos-based communication systems have been inspired by non-coherent detection, which does not involve any complex synchronization mechanism between the transmitter and the receiver.

\section{Conclussion}

Communication techniques focused on chaotic processes have been the focus of intense research since Carroll and Pecora proposed their approach to synchronize chaos in 1991. We study a large number of related research, such as chaotic coding, chaotic modulation/demodulation, and multiple-access communication schemes, in this paper.

His survey provides a strong, transparent, and straightforward entry point into the topic. Furthermore, we present a classification for various modulation techniques as well as a detailed discussion of their benefits and drawbacks. We also go over multiple-access methods and chaos-based non-coherent detection techniques in detail. Finally, the aim of this review is to provide readers with useful tools for comprehending current research contributions in the rapidly expanding field of chaos communication systems. This will help to stimulate further research into the design of nextgeneration chaos-based wireless communication systems.

\section{References}

Afraimovich, V. S., Verichev, N. N., \& Rabinovich, M. I. (1986). Stochastic synchronization of oscillation in dissipative systems. Radiophys. Quantum Electron, 29(9), 795-803.

Alligood, K. T., Sauer, T. D., \& Yorke, J. A. (1996). CHAOS: An Introduction to Dynamical Systems. Heidelberg, Germany: Springer-Verlag.

Bailey, J. P., Beal, A. N., Dean, R. N., Hamilton, M. C., \& Tugnait, J. K. (2014). High-frequency reverse-time chaos generation using digital chaotic maps. Electron. Lett, 50(23), 1683-1685.

Beirami, A., Nejati, H., \& Ali, W. H. (2012). Zigzag map: A variability-aware discrete-time chaotic-map truly random number generator. Electron. Lett, 48(24), 1537-1538.

Berber, S., \& Feng, S. (2013). Chaos-based physical layer design for WSN applications, in Proc. CIRCOM, 2, $157-162$.

Chen, S. Y., Xi, F., \& Liu, Z.(2014). Chaotic analogue-to-information conversion with chaotic state modulation. IET Signal Process, 8(4), 373-380.

Chua, L. O. (1980). Dynamic nonlinear networks: State-of-the-art. IEEE Trans. Circuits Syst, 27 (11), 1059-1087.

Chua, L. O., \& Lin, G.-N. (1990). Canonical realization of Chua's circuit family. IEEE Trans. Circuits Syst., 37 (7), $885-902$.

Cuomo, K.M., \& Oppenheim, A. V.(1993). Circuit implementation of synchronized chaos with applications to communications. Phys. Rev. Lett, 71, 65-68.

Cuomo, K.M., Oppenheim, A. V., \& Strogatz, S. H. (1993). Synchronization of Lorenz-based chaotic circuits with applications to communications. IEEE Trans. Circuits Syst. I, Fundam. Theory Appl, 40(10), 626-633.

Dedieu, H., Kennedy, M. P., \& Hasler, M. Chaos shift keying: Modulation and demodulation of a chaotic carrier using selfsynchronizing

Delgado-Restituto, M., \& Rodriguez-Vazquez, A. (2001). Mixed-signal map-congurable integrated chaos generator for chaotic communications. IEEE Trans. Circuits Syst. I, Fundam. Theory Appl, 48(12), 1462-1474.

Devaney, R. L.(2003). An Introduction to Chaotic Dynamical Systems. Westview Pr (Short Disc).

Fang, Y., Xu, L., Wang, L \& Chen, G. (2013). Performance of MIMO relay DCSK-CD systems over Nakagami fading channels. IEEE Trans. Circuits Syst. I, Reg. Papers, 60(3), 757-767. 
Feng, J.C., \& Tse, C. K. (2001). On-line adaptive chaotic demodulator based on radial-basis-function neural networks. Phys. Rev. E, 63, 026202.

Fujisaka, H., \& Yamada, T. (1983). Stability theory of synchronized motion in coupled-oscillator systems. Prog. Theor. Phys, 69(1), 32-47.

Giard, P., Kaddoum, G., Gagnon, F., \& Thibeault, C. (2012). FPGA imple-mentation and evaluation of discrete-time chaotic generators circuits. in Proc. 38th IEEE Annu. Conf. Ind. Electron. Soc. (IECON). 3221-3224.

Hasler, M., \& Schimming, T. (2002). Optimal and suboptimal chaos receivers. Proc. IEEE, 90(5), 733-746.

Jovic, B. (2015). Chaos-based BPSK communication system. Electron. Lett, 51(8), 630-632.

Kaddoum, G., Richardson, F., \& Gagnon, F. (2013). Design and analysis of a multi-carrier differential chaos shift keying communication system. IEEE Trans. Commun, 61(8), 3281-3291).

Kennedy, M. P., \& Kolumbán, G. Kis, G. and Jákó, Z. (2000). Perfor-mance evaluation of FM-DCSK modulation in multipath environments. IEEE Trans. Circuits Syst. I, Fundam. Theory Appl, 47(12), 1702-1711,

Kennedy, M. P., Rovatti, R., \& Setti, G. (2000) Chaotic Electronics in Telecommunications. London, U.K.: CRC Press, Chua's circuits. IEEE Trans. Circuits Syst. II, Analog Digit. Signal

Kis, G., Jákó, Z., Kennedy, M. P., \& Kolumbán, G. (1998). Chaotic communications without synchronization. in Proc. 6th IEE Conf. Telecommun. 49-53.

Kolumbán, G., Kennedy, M. P., \& Chua, L. O. (1997). The role of synchronization in digital communications using chaos. I. Fundamentals of digital communications. IEEE Trans. Circuits Syst. I, Fundam, Theory Appl, 44(10), 927-936.

Kolumbán, G., Vizvári, G. K., Schwarz, W., \& Abel, S. (1996). Differential chaos shift keying: A robust coding for chaos communication. in Proc.Int. Workshop Non-Linear Dyn. Electron. Syst. (NDES), Seville, Spain. 92-97.

Kurian, A. P., Puthusserypady, S., \& Htut, S. M. (2005). Performance enhancement of DS/CDMA system using chaotic complex spreading sequence. IEEE Trans. Wireless Commuz, 4(3), 984-989.

Lau, F. C. M., \& Tse, C. K. (2003). Chaos-Based Digital Communication Systems. Heidelberg, Germany: Springer-Verlag.

Lee, C., \& Williams, D.B., (1997). Generalized iterative methods for enhancing contaminated chaotic signals. IEEE Trans. Circuits Syst. I, Fundam. Theory Appl, 44(6), 501-512.

Li, Z., \& Chen, G. (2006). Global synchronization and asymptotic stability of complex dynamical networks. IEEE Trans. Circuits Syst. II, Exp. Briefs, 53(1), 28-33.

Liao, T. L., \& Huang, N. S.(1999). An observer-based approach for chaotic synchronization with applications to secure communications. IEEE Trans. Circuits Syst. I, Fundam. Theory Appl, 46(9), 1144-1150.

Luca, M. B., Azou, S., Burel, G., \& Serbanescu, A. (2006). On exact Kalman ltering of polynomial system. IEEE Trans. Circuits Syst. I, Reg. Papers, vol. 5(6), 1329-1340.

Lynnyk, V., \& Elikovský, S. (2010). On the anti-synchronization detection for the generalized Lorenz system and its applications to secure encryption. Kybernetika, 46 (1), 1-18.

Mahmoud, E. E. (2014). Complex complete synchronization of two nonidentical hyperchaotic complex nonlinear systems. Math. Methods Appl. Sci., 37(3), 321-328.

Mazzini, G., Setti, G, \& Rovatti, R. (1997) Chaotic complex spreading sequences for asynchronous DS-CDMAPart I: System modeling and results. IEEE Trans. Circuits Syst. I, Fundam. Theory Appl, 44 (10), 937-947.

Mbe, E. S. K., Fotsin, H. B., Kengne, J \& Woafo, P. (2014). Parameters estimation based adaptive generalized projective synchronization (GPS) of chaotic Chua's circuit with application to chaos communication by parametric modulation. Chaos, Solitons Fractals, 61, pp. 27-37.

Milanovic, V. M., \& Zaghloul, M. E. (1996). Improved masking algorithm for chaotic communications systems. Electron. Lett, 
$32(1), 11-12$.

Mobayen, S., Fekih, A., Vaidyanathan, S., \& Sambas, A. (2021). Chameleon Chaotic Systems With Quadratic Nonlinearities: An Adaptive Finite-Time Sliding Mode Control Approach and Circuit Simulation. IEEE Access, 9, 64558-64573.

Nejati, H., Beirami, A., \& Ali, W. H. (2012). Discrete-time chaotic-map truly random number generators: Design, implementation, and variability analysis of the zigzag map. Analog Integr. Circuits Signal Process, 73(1), 363-374.

Pecora, L. M., \& Carroll, T. L. (1990). Synchronization in chaotic systems. Phys. Rev. Lett., 64, 821-823.

Sambas, A., Mada Sanjaya, W. S., \& Mamat, M. (2015). Bidirectional Coupling Scheme of Chaotic Systems and its Application in Secure Communication System. Journal of Engineering Science and Technology Review, 8(2), 89-95.

Sambas, A., Vaidyanathan, S., Tlelo-Cuautle, E., Zhang, S., Guillen-Fernandez, O., Hidayat, Y., \& Gundara, G. (2019). A novel chaotic system with two circles of equilibrium points: multistability, electronic circuit and FPGA realization. Electronics, 8(11), 1211.

Sambas, A., Vaidyanathan, S., Tlelo-Cuautle, E., Abd-El-Atty, B., Abd El-Latif, A. A., Guillén-Fernández, O., Hidayat, Y \& Gundara, G. (2020). A 3-D multi-stable system with a peanut-shaped equilibrium curve: Circuit design, FPGA realization, and an application to image encryption. IEEE Access, 8, 137116-137132.

Sambas, A., Vaidyanathan, S., Bonny, T., Zhang, S., Hidayat, Y., Gundara, G., \& Mamat, M. (2021). Mathematical Model and FPGA Realization of a Multi-Stable Chaotic Dynamical System with a Closed Butterfly-Like Curve of Equilibrium Points. Applied Sciences, 11(2), 788.

Shannon, C. E. (1998). Communication in the presence of noise. Proc. IEEE, 86(2), 447- 457.

Silva, C. P., \& Young, A. M. (2000). Introduction to chaos-based commu-nications and signal processing. in Proc. IEEE Aerosp. Conf., 1, 279-299.

Soriano, D. C., Attux, R.,. Romano, J. M. T.,. Loiola, M. B., \& Suyama, R. (2011). Denoising chaotic time series using an evolutionary state estimation approach. in Proc. IEEE Symp. Comput. Intell. Control Autom. (CICA), 116-122.

Suykens, J. A. K., Curran, P. F., \& Chua, L. O. (1997). Master-slave synchronization using dynamic output feedback. Int. J. Bifurcation Chaos 7(3), 671-679.

Vaidyanathan, S., Azar, A. T., Rajagopal, K., Sambas, A., Kacar, S., \& Cavusoglu, U. (2018). A new hyperchaotic temperature fluctuations model, its circuit simulation, FPGA implementation and an application to image encryption. International Journal of Simulation and Process Modelling, 13(3), 281-296.

Vaidyanathan, S., Dolvis, L. G., Jacques, K., Lien, C. H., \& Sambas, A. (2019). A new five-dimensional four-wing hyperchaotic system with hidden attractor, its electronic circuit realisation and synchronisation via integral sliding mode control. International Journal of Modelling, Identification and Control, 32(1), 30-45.

Vaidyanathan, S., Tlelo-Cuautle, E., Sambas, A., Dolvis, L. G., Guillén-Fernández, O., \& Idowu, B. A. (2020). A new multistable hyperjerk dynamical system with self-excited chaotic attractor, its complete synchronisation via backstepping control, circuit simulation and FPGA implementation. International Journal of Modelling, Identification and Control, 35(3), 177190.

Vaidyanathan, S., Sambas, A., Azar, A. T., Rana, K. P. S., \& Kumar, V. (2021). A new 5-D hyperchaotic four-wing system with multistability and hidden attractor, its backstepping control, and circuit simulation. In Backstepping Control of Nonlinear Dynamical Systems (pp. 115-138). Academic Press.

Vali, R., Berber, S. M \& Nguang, S. K. (2010). Effect of Rayleigh fading on non-coherent sequence synchronization for multiuser chaos based DS-CDMA. Signal Process, 90(6), 1924-1939.

Vali, R., Berber, S., \& Nguang, S. K. (2012). Accurate derivation of chaos-based acquisition performance in a fading channel. IEEE Trans. Wireless Commun, 11(2), 722-731.

Vali, R., Berber, S., \& Nguang, S. K. (2012). Analysis of chaos-based code tracking using chaotic correlation statistics. IEEE Trans. Circuits Syst. I, Reg. Papers, 59(4), 796-805. 
Vitali, S., Rovatti, R., \& Setti, G. (2006). Improving PA efciency by chaos-based spreading in multicarrier DS-CDMA systems. in Proc. IEEE Int Symp. Circuits Syst. (ISCAS), 1194-1198.

Wu, G. C., \& Baleanu, D. (2014). Chaos synchronization of the discrete fractional logistic map. Signal Process, 102, 96-99.

Wu, Y., Zhou, Y., \& Bao, L. (2014). Discrete wheel-switching chaotic system and applications. IEEE Trans. Circuits Syst. I, Reg. Papers, 61(12), 3469- 3477.

Xia, Y., Tse, C. K., \& Lau, F. C. M (2004). Performance of differential chaos-shift-keying digital communication systems over a multipath fading channel with delay spread. IEEE Trans. Circuits Syst. II, Exp. Briefs, 51(12), 680-684.

Yalçin, M. E., Suykens, J. A. K., \& Vandewalle, J. (2001). Master slave synchronization of Lur'e systems with time-delay. Int. J. Bifurcation Chaos, 11(6), 1707-1722.

Yang, X., Cao, J., \& Lu, J. (2012). Stochastic synchronization of complex networks with non-identical nodes via hybrid adaptive and impulsive control. IEEE Trans. Circuits Syst. I, Reg. Papers, 59(2), 371-384.

Yu, J., \& Yao, Y. D. (2005). Detection performance of chaotic spreading LPI waveforms. IEEE Trans. Wireless Commun, 4(2), 390-396.

Yu, J., Hu, C., Jiang, H., \& Fan, X. (2014). Projective synchronization for fractional neural networks. Neural Netw, vol. 49 87-95.

Zhou, B. B., \& Roy, R. (2007). Isochronal synchrony and bidirectional communication with delay-coupled nonlinear oscillators. Phys. Rev. E, 75 . 